\title{
СПЕКТР ПОГЛОЩЕНИЯ ЭКСИТОНА. ОБЩИЕ ФОРМУЛЫ
}

\author{
(Представил В. Хижняков)
}

В опубликованных к настоящему времени теоретических работах, посвященных изучению спектра поглощения экситона, взаимодействующего с фононами, вычисления основывались либо на предположениях о малости определенных параметров задачи, оправданных для некоторых типов кристаллов (см., напр., $\left.\left[{ }^{1-4}\right]\right)$, либо на использовании ряда предположений, применимость которых требует дополнительной проверки (напр., приближение динамического когерентного потенциала в [ [5], адиабатическое приближение в $\left.\left[{ }^{6}\right]\right)$.

Целью данной работы является получение формул, описывающих спектр поглощения экситона в идеальном кристалле при $T=0$, в виде, позволяющем создать алгоритм решения этой задачи, не использующий предложений типа перечисленных выше. Разумеется, для реализации этого алгоритма потребуется достаточно мощная ЭВМ. Основой рассмотрения является метод тридиагонализации гамильтониана, ранее успешно использовавшийся для вычислений плотностей электронных и фононных состояний идеальных кристаллов, поверхностей и неупорядоченных систем (см. $[7,8]$ и ссылки там же).

Нормированный на единицу спектр поглощения экситона при $T=0$ может быть выражен через функцию Грина следующим образом:

$$
A(\Omega)=-\pi^{-1} \operatorname{Im} G(\vec{x}, \Omega+i \gamma)=-\pi^{-1} \operatorname{Im}\left\langle 0\left|(\Omega+i \gamma-H)^{-1}\right| 0\right\rangle,
$$

где $\boldsymbol{x}$ - волновой вектор фотона, который далее будем полагать равным нулю, $\gamma \rightarrow+0$, символом $|0\rangle$ обозначено состояние $\underset{x}{a_{\vec{x}}^{+}}|\mathrm{vac}\rangle, a_{\vec{x}}^{+}-$оператор рождения экситона с волновым вектором $\vec{x}(\approx 0)$ (в данной работе рассматривается невырожденная экситонная зона, см. (2)), |vac $\rangle$ - вакуумное состояние экситонов и фононов. Гамильтониан кристалла

$$
H=\sum_{\mathbf{k}} \varepsilon(\mathbf{k}) a_{\mathbf{k}}^{+} a_{\mathbf{k}}+\sum_{\mathbf{k}} \omega(\mathbf{k}) b_{\mathbf{k}}^{+} b_{\mathbf{k}}+\sum_{\mathbf{k}, \mathbf{k}^{\prime}} v(\mathbf{k}) a_{\mathbf{k}+\mathbf{k}^{\prime}}^{+} a_{\mathbf{k}^{\prime}}\left(b_{\mathbf{k}}+b_{-\mathbf{k}}^{+}\right),
$$

где $\varepsilon(\mathbf{k})$ - энергия экситона с волновьм вєктором $\mathbf{k}, b_{\mathrm{k}}^{+}$- оператор рождения фонона с волновым вектором $\mathbf{k}$ и частстой $\omega(\mathbf{k}), v(\mathbf{k})-$ константа экситон-фононного взаимодействия.

Для вычисления функции Грина в (1) достаточно знать гамильтониан (2) на подпространстве векторов состояния, получаемых при многократном действии (2) на вектор $|0\rangle$. Представим его в этом подпространстве в трехдиагональном виде

$$
H=\sum_{n=0}^{\infty} E_{n}|n\rangle\left\langle n\left|+\sum_{n, n^{\prime}=0}^{\infty} V_{n, n^{\prime}}\right| n\right\rangle\left\langle n^{\prime}\right| .
$$


Шітрих во второй сумме означает, что $n^{\prime}=n \pm 1$. Состо̆яния $|n\rangle$ предполагаются ортогональными и ортонормированными: $\left\langle n \mid n^{\prime}\right\rangle=\delta_{n, n^{\prime}}$. Из эрмитовости гамильтониана (3) следует: $V_{n, n+1}=V_{n+1, n}$ (величины $V_{n+1, n}$ для гамильтониана (2) можно выбрать действительными). Действуя гамильтонианами (2) и (3) на вектор $|n\rangle$, находим рекуррентные формулы, определяющие базис $\{|n\rangle\}$ :

$$
\begin{aligned}
& |1\rangle=V_{10}^{-1}\left(H-E_{0}\right)|0\rangle, \\
& |n+1\rangle=V_{n+1, n}^{-1}\left[\left(H-E_{n}\right)|n\rangle-V_{n, n-1}|n-1\rangle\right], \quad n \geqslant 1, \\
& \quad E_{n}=\langle n|H| n\rangle, \quad V_{n+1, n}=\langle n+1|H| n\rangle .
\end{aligned}
$$

Используем операторные тождества

$$
\left.\begin{array}{l}
R=R_{0}+R_{0} H_{1} R \\
\Lambda R=\Lambda R_{\Lambda} \Lambda+\Lambda R_{\Lambda} \Lambda H_{1}(1-\Lambda) R,
\end{array}\right\}
$$

$R=(z-H)^{-1}, H=H_{0}+H_{1}, R_{0}=\left(z-H_{0}\right)^{-1}, \Lambda=1-|a\rangle\langle a|, R_{\Lambda}=$ $=\left(z-H_{0}-\Lambda H_{1} \Lambda\right)^{-1} \quad$ (доказательство второго из тождеств (6) можно найти в $\left.\left[{ }^{9}\right]\right)$. Полагая вначале $|a\rangle=|0\rangle$ и принимая за $H_{0}$ первое слагаемое в гамильтониане (3), а за $H_{1}-$ второе, находим

$$
\begin{gathered}
G(z)=\left[z-E_{0}-\Sigma(z)\right]^{-1}, \\
\Sigma(z)=\left\langle 0\left|H_{1} \Lambda_{0}\left(z-H_{0}-\Lambda_{0} H_{1} \Lambda_{0}\right)^{-1} \Lambda_{0} H_{1}\right| 0\right\rangle= \\
=V_{10}^{2}\left\langle 1\left|\left(z-H_{0}-\Lambda_{0} H_{1} \Lambda_{0}\right)^{-1}\right| 1\right\rangle .
\end{gathered}
$$

Как видим, функция Грина (1) и ее массовый оператор $\Sigma(z)$ имеют сходное строение с той лишь разницей, что в последнем вместо $|0\rangle$ и $H_{1}$ фигурируют $|1\rangle$ и $\Lambda_{0} H_{1} \Lambda_{0}$. Операторы проектирования в последнем выражении исключают из второй суммы в (3) два слагаемых, содержащих вектора $|0\rangle$ и $\langle 0|$. Полагая $|a\rangle=|1\rangle, H^{\prime}=\Lambda_{0} H_{1} \Lambda_{0}$ и, вновь используя тождества (6), находим:

$$
\begin{aligned}
& \Sigma(z)=V_{10}^{2}\left[z-E_{1}-\Sigma^{\prime}(z)\right]^{-1} \\
& \Sigma^{\prime}(z)=V_{21}^{2}\left\langle 2\left|\left(z-H_{0}-\Lambda_{1} H^{\prime}{ }_{1} \Lambda_{1}\right)^{-1}\right| 2\right\rangle
\end{aligned}
$$

Продолжая в том же духе, приходим к представлению функции Грина в виде цепной дроби:

$$
G(z)=\frac{1}{z-E_{0}-\frac{V_{10}^{2}}{z-E_{1}-\frac{V_{21}^{2}}{z-E_{2}-\frac{V_{32}^{2}}{\vdots}}}}
$$

(иной вывод формулы (7) см. в $\left[{ }^{7}\right]$ ). Обрывая дробь на $n-м$ звене, т. е. аппроксимируя цепную дробь $n$-й подходящей дробью $\left[{ }^{10}\right]$, мы представляем спектр поглощения (1) в виде $n \delta$-функций.

Вернемся к гамильтониану (2). Представим векторы $|n\rangle$, фигурирующие в (3), в виде 


$$
|n\rangle=\sum_{v=0}^{n} \sum_{\mathbf{k}_{1}, \ldots \mathbf{k}_{v}} C_{n, v}\left(\mathbf{k}_{1}, \ldots \mathbf{k}_{v}\right) b_{\mathbf{k}_{1}, \ldots}^{+} \ldots b_{\mathbf{k}_{v}}^{+} a_{\mathbf{K}}^{+}|v a c\rangle
$$

$\mathbf{K}+\sum_{i=1}^{v} \mathbf{k}_{i}=0$ (подпространство $\{|n\rangle\}$, содержащее вектор $|0\rangle$, состоит из векторов, отвечающих нулевому суммарному волновому вектору). Из условий ортогональности и нормировки, используя теорему ВикаБлоха-Доминисиса [ $\left.{ }^{11}\right]$, находим:

$$
\sum_{v=0}^{\min \left(n, n^{\prime}\right)} v ! \sum_{\mathrm{k}_{1}, \ldots \mathrm{k}_{v}} C_{n^{\prime}, v}\left(\mathrm{k}_{1} ; \ldots \boldsymbol{k}_{v}\right) C_{n, v}\left(\mathrm{k}_{1}, \ldots \mathbf{k}_{v}\right)=\delta_{n, n^{\prime}}
$$

Подставляя (8) в (4), действуя слева вектором 〈vac $\mid a_{\mathbf{K}} b_{\mathbf{k}_{1}}, \ldots b_{\mathbf{k}_{v}}$ и используя теорему Вика-Блоха-Доминисиса, получаем рекуррентные формулы в виде

$$
\begin{gathered}
C_{n+1,0}=V_{n+1, n}^{-1}\left\{\left[\varepsilon(0)-E_{n}\right] C_{n, 0}+\theta(n-1) \sum_{\mathbf{k}} v(\mathbf{k}) C_{n, 1}(\mathbf{k})-\right. \\
\left.-\theta(n-1) V_{n, n-1} C_{n-1,0}\right\}, \\
C_{n+1, v}\left(\mathbf{k}_{1}, \ldots \mathbf{k}_{v}\right)=V_{n+1, n}^{-1}\left\{\theta(n-v)\left[\varepsilon\left(\sum_{i=1}^{v} \mathbf{k}_{i}\right)+\sum_{i=1}^{v} \omega\left(\mathbf{k}_{i}\right)-E_{n}\right] \times\right. \\
\times C_{n, v}\left(\mathbf{k}_{1}, \ldots \mathbf{k}_{v}\right)+\theta(n-v-1)(v+1) \sum_{\mathbf{k}_{v+1} v\left(\mathbf{k}_{v+1}\right) C_{n, v+1}\left(\mathbf{k}_{1}, \ldots \mathbf{k}_{v+1}\right)+} \\
+\frac{1}{v} \sum_{i=1}^{v} v\left(\mathbf{k}_{i}\right) C_{n, v-1}\left(\mathbf{k}_{1}, \ldots \mathbf{k}_{i-1} \mathbf{k}_{i+1}, \ldots \mathbf{k}_{v}\right)- \\
\left.\quad-\theta(n-v-1) V_{n, n-1} C_{n-1, v}\left(\mathbf{k}_{1}, \ldots \mathbf{k}_{v}\right)\right\}, \\
v \geqslant 1 .
\end{gathered}
$$

$\theta(m)=0$ при $m<0, \theta(m)=1$ при $m \geqslant 0$. Рекурсия начинается со значения $C_{00}=1$.

Проведем преобразование Фурье формул -(10) и (11), для чего введем фурье-образ функций $C_{n, v}\left(\mathbf{k}_{1}, \ldots \mathbf{k}_{v}\right)$ :

$D_{n, 0}=C_{n, 0}$,

$D_{n, v}\left(\mathbf{m}_{1}, \ldots \mathbf{m}_{v}\right)=N-v / 2 \sum_{\mathbf{k}_{1}, \ldots \mathbf{k}_{v}} \exp \left(-i \sum_{j=1}^{v} \mathbf{m}_{j} \cdot \mathbf{k}_{j}\right) C_{n, v}\left(\mathbf{k}_{1}, \ldots \mathbf{k}_{v}\right), v \geqslant 1$,

$N$ - число узлов периодической области. Из условий (9) следует, что

$$
\sum_{v=0}^{\min \left(n, n^{\prime}\right)} v ! \sum_{\mathbf{m}_{1}, \ldots \mathrm{m}_{v}} D_{n^{\prime}, v}\left(\mathbf{m}_{1}, \ldots \mathbf{m}_{v}\right) D_{n, v}\left(\mathbf{m}_{1}, \ldots \mathbf{m}_{v}\right)=\delta_{n, n^{\prime}}
$$

Полученные выше формулы справедливы для любых законов дисперсии $\varepsilon(\mathbf{k}), v(\mathbf{k})$ и $\omega(\mathbf{k})$. В данной работе положим $v(\mathbf{k})=v, \omega(\mathbf{k})=\omega$, что отвечает взаимодействию экситона с неполярными оптическими колебаниями; считая кристалл простым кубическим, положим

$$
\varepsilon(\mathbf{k})=-\frac{B}{6}\left[\cos \left(k_{x}\right)+\cos \left(k_{y}\right)+\cos \left(k_{z}\right)\right],
$$

где $k_{i}$ - декартовы координаты вектора k. Из (10) и (11) находим: 


$$
\begin{aligned}
D_{n+1,0}=V_{n+1, n}^{-1} & \left\{-\left(\frac{\bar{B}}{2}+E_{n}\right) D_{n, 0}+\theta(n-1) v N^{1 / 2} D_{n, 1}(0)-\right. \\
& \left.-\theta(n-1) V_{n, n-1} D_{n-1,0}\right\}, .
\end{aligned}
$$

$$
\begin{gathered}
D_{n+1, v}\left(\mathrm{~m}_{1}, \ldots \mathrm{m}_{v}\right)=V_{n+1, n}^{-1}\left\{\theta(n-v)\left(v \omega-E_{n}\right) D_{n, v}\left(\mathbf{m}_{1}, \ldots \mathbf{m}_{v}\right)-\right. \\
-\theta(n-v) \frac{B}{12} \sum_{\mathbf{a}} D_{n, v}\left(\mathbf{m}_{1}-\mathbf{a}, \ldots \mathbf{m}_{v}-\mathbf{a}\right)+\theta(n-v-1)(v+1) \times
\end{gathered}
$$

$$
X v N^{1 / 2} D_{n, v+1}\left(\mathbf{m}_{1}, \ldots \mathbf{m}_{v} ; 0\right)+\frac{v N^{1 / 2}}{v} \sum_{i=1}^{v} \delta_{\mathrm{m}_{i}, 0} D_{n, v-1} X
$$

$\left.\times\left(\mathbf{m}_{1}, \ldots \mathbf{m}_{i-1}, \mathbf{m}_{i+1}, \ldots \mathbf{m}_{v}\right)-\theta(n-v-1) V_{n, n-1} D_{n-1, v}\left(\mathbf{m}_{1}, \ldots \mathbf{m}_{v}\right)\right\}$,

$$
v \geqslant 1 \text {. }
$$

В (15) суммирование по а производится по 6 единичным векторам решетки (т. е. $\mathbf{a}=(1,0,0),(-1,0,0)$ и т. д.). Рекурсия стартует со значения $D_{00}=1$. Пользуясь условиями (13), из (14) и (15) получаем формулы для величин $E_{n}$ и $V_{n, n-1}^{2}$, входящих в (7):

$$
\begin{aligned}
& E_{n}=\omega \sum_{v=0}^{n} v ! v \sum_{\mathrm{m}_{1}, \ldots \mathrm{m}_{v}}^{\sum} D_{n, v}^{2}\left(\mathrm{~m}_{1}, \ldots \mathrm{m}_{v}\right)-\frac{B}{12} \sum_{\mathbf{a}} \sum_{v=0}^{n} v ! \sum_{\mathrm{m}_{1}, \ldots \mathrm{m}_{v}}^{\sum} D_{n, v}\left(\mathrm{~m}_{1}, \ldots \mathrm{m}_{v}\right) \times \\
& \times D_{n, v}\left(\mathbf{m}_{1}-\mathbf{a}, \ldots \mathbf{m}_{v}-\mathbf{a}\right)+2 v N^{1 / 2} \sum_{v=0}^{n-1}(v+1) ! \sum_{\mathbf{m}_{1}, \ldots \mathrm{m}_{v}} D_{n, v}\left(\mathbf{m}_{1} ; \ldots \mathbf{m}_{v}\right) \times \\
& +D_{n, v+1}\left(\mathbf{m}_{1}, \ldots \mathbf{m}_{v}, 0\right) \text {, } \\
& V_{n, n-1}^{2}=\omega \sum_{v=0}^{n-1} v ! v \sum_{\mathrm{m}_{1}, \ldots \mathrm{m}_{v}} D_{n-1, v}\left(\mathbf{m}_{1}, \ldots \mathbf{m}_{v}\right)\left[V_{n, n-1} D_{n, v}\left(\mathbf{m}_{1}, \ldots \mathbf{m}_{v}\right)\right]- \\
& -\frac{B}{12} \sum_{\mathbf{a}} \sum_{v=0}^{n-1} v ! \sum_{\mathrm{m}_{1}, \ldots \mathrm{m}_{v}} D_{n-1, v}\left(\mathrm{~m}_{1}, \ldots \mathrm{m}_{v}\right)\left[V_{n, n-1} D_{n, v}\left(\mathbf{m}_{1}-\mathbf{a}, \ldots \mathbf{m}_{v}-\mathbf{a}\right)\right]+ \\
& +v N^{1 / 2} \sum_{v=0}^{n-1}(v+1) ! \sum_{\mathrm{m}_{1}, \ldots \mathrm{m}_{v}} D_{n-1, v}\left(\mathbf{m}_{1}, \ldots \mathbf{m}_{v}\right)\left[V_{n, n-1} D_{n, v+1}\left(\mathbf{m}_{1}, \ldots \mathbf{m}_{v}, 0\right)\right]+ \\
& +v N^{1 / 2} \sum_{v=1}^{n-1} v ! \sum_{\mathrm{m}_{1}, \ldots \mathrm{m}_{v}} D_{n-1, v}\left(\mathrm{~m}_{1}, \ldots \mathrm{m}_{v-1}, 0\right)\left[V_{n, n-1} D_{n, v-1}\left(\mathrm{~m}_{1}, \ldots \mathbf{m}_{v-1}\right)\right]
\end{aligned}
$$

Алгоритм вычисления $E_{n}$ и $V_{n, n-1}^{2}$ выглядит следующим образом. Используя стартующую величину $D_{00}=1$, вычисляем по формуле (16) $E_{0}$

$$
E_{0}=-\frac{B}{12} \sum_{\mathrm{a}} D_{00}^{2}=-\frac{B}{2} .
$$

По формулам (14) и (15) находим $V_{10} D_{10}$ и $V_{10} D_{11}(\mathrm{~m})$

$$
V_{10} D_{10}=-\left(\frac{B}{2}+E_{0}\right) D_{00}=0, \quad V_{10} D_{11}(\mathrm{~m})=v N^{1 / 2} \delta_{\mathrm{m}, 0} D_{00}
$$

(отметим, что $D_{n 0}=0$ при $n \geqslant 1$ ввиду условий ортогональности (13); эти условия позволяют получить бесконечную последовательность ра- 
венств, которую также можно использовать для нахождения величин $E_{n}$ и $\left.V_{n, n-1}^{2}\right)$. Подставляя найденные величины в (17), находим $V_{10}^{2}$

$$
V_{10}^{2}=v N^{1 / 2} D_{00}\left[V_{10} D_{10}(0)\right]=v^{2} N .
$$

После этого можем определить $D_{11}(\mathrm{~m})$ и, подставив ее в $(16)$, найти $E_{1}$

$$
E_{1}=\omega \sum_{\mathrm{m}} D_{11}^{2}(\mathrm{~m})=\omega .
$$

Цикл повторяется: по формуле (15), используя полученные выше величины, находим

$$
\begin{aligned}
& V_{21} D_{21}(\mathrm{~m})=-\frac{B}{12} \sum_{\mathbf{a}} D_{11}(\mathrm{~m}-\mathbf{a})=-\frac{B}{12} \underset{\mathbf{a}}{\sum} \delta_{\mathrm{m}, \mathrm{a}}, \\
& V_{21} D_{22}\left(\mathrm{~m}_{1}, \mathrm{~m}_{2}\right)=\frac{v N^{1 / 2}}{2} \sum_{i=1}^{2} \delta_{\mathrm{m}_{i}, 0} D_{11}\left(\mathrm{~m}_{i^{\prime}}\right)=v N^{1 / 2} \delta_{\mathrm{m}_{1}, 0} \delta_{\mathrm{m}_{2}, 0}, \quad i^{\prime} \neq i, \quad i^{\prime}=1,2,
\end{aligned}
$$

вычисляем $V_{21}^{2}, E_{2}$ и т. д. Как следует из формул (14) и (15), в памяти ЭВМ должна храниться информация лишь о двух наборах величин: $D_{n, v}$ и $D_{n-1, v}$, что достаточно для вычисления $V_{n+1, n} D_{n+1, v}$. С ростом $n$ возрастают как радиусы сфер, в которых расположены концы векторов $\mathbf{m}_{i}$ $\left(i=(1, \ldots v)\right.$, отвечающих ненулевым $D_{n, v}\left(\mathrm{~m}_{1}, \ldots \mathrm{m}_{\mathrm{v}}\right)$, так и числа $v \leqslant n$. Поэтому число величин $D_{n, v}$, требующих запоминания, довольно быстро растет. Ситуация несколько улучшается благодаря их симметрии: по определению они симметричны относительно перестановки любой пары векторов $\mathbf{m}_{1}, \ldots \mathbf{m}_{v}$ (см. (8) и (12)), а также инварианты относительно одновременного действия на все векторы $\mathrm{m}_{i}$ операций симметрии группы $O_{h}$. Это обстоятельство значительно сокращает требуемый объем памяти.

В [5] функция Грина приводится к виду, похожему на формулу (7). Однако там этот результат получен с использованием приближения динамического когерентного потенциала, тогда как наш вывод точен. Как следствие, аналоги величин $V_{n, n-1}^{2}$ в [ $\left.{ }^{5}\right]$ равны $n v^{2} N$ в наших обозначениях, что соответствует локализованному возбуждению. В нашем расчете эти величины имеют более сложный вид уже при $n=2$ (напр., $V_{21}^{2}=$ $\left.=2 v^{2} N+B^{2} / 24\right)$.

Таким образом, формулы (14)-(17) позволяют вычислить спектр поглощения экситона, взаимодействующего с фононами, не привлекая допущений о малости какого-либо параметра или каких-либо иных предположений. Результаты, полученные при реализации описанного выше алгоритма, будут опубликованы в следующей статье.

Автор признателен В. Хижнякову за полезное обсуждение.

\section{Л ИТЕ РА Т У РА}

1. Москаленко С. А., Шлиелюк М. И., Чиник Б. И. В кн.: Экситонное поглощение света и кинетика экситонов в полупроводниках. Кишинев, АН МССР, 1968, $3-27$.

2. Федосеев В. Г. Препринты FAI-5 и FAI-6, Тарту, 1970.

3. Hizhnyakov, V. V., Sherman, A. V. Phys status solidi (b), 92, № $1,177-183$ (1979).

4. Броуде В. Л., Рашба Э. И., Шека Е. Ф. Спектроскопия молекулярных экситонов. М., «Энергонздат», 1981.

5. Sumi, H. J. Phys. Soc. Japan, 36, № 3, 770-779 (1974); 38, № 3, 825-835 (1975). 
6. Schreiber, M., Toyozawa, Yu. J. Phys. Soc. Japan, 51, № 5, 1528-1536, 1537-1543, $1544-1550$ (1982).

7. Haydock, R. Solid State Phys., 35, 215-294 (1980).

8. Kelly, M. J. Solid State Phys., 35, 295-383 (1980).

9. Кролль Н. В кн.: Квантовая оптика и квантовая радиофизика. М., «Мир», 1966, $9-89$.

10. Хованский А. Н. Приложение цепных дробей и их обобщений к вопросам приближенного анализа. М., ГИТТЛ, 1956.

11. Тябликов С. В. Методы квантовой теории магнетизма. М., «Наука», 1975.

Ннститут физики

Академии наук Эстонской ССР
Поступила в редакцию $23 /$ X 1984

\section{A. SERMAN}

\section{EKSITONI NEELDUMISSPEKTER. OLDISED VALEMID}

On vaadeldud foononitega vastasmõjus oleva eksitoni neeldumisspektrit. Greeni funktsioon spektri määramiseks on teisendatud ahelmurruks (7), mille koefitsiendid on leitud rekursioonimeetodil (vt. (14)-(17)). Viimane võimaldab määrata suvaliste parameetrite spektri täpselt, ilma adiabaatilist lähendust kasutamata.

\section{A. SHERMAN}

\section{EXCITON ABSORPTION SPECTRUM. GENERAL FORMULAE}

An absorption spectrum of an exciton interacting with phonons is considered. The Green function determining the spectrum is transformed unto a continued fraction (7), coefficients of which are computed by the recursion method (see Eqs (14)-(17)). This allows one to compute the spectrum exactly for the arbitrary parameters $B$, exciton bandwidth, $v$, vibronic constant, $\omega$, phonon frequency, without making use of the adiabatic aproximation, 